\title{
COVID-19: ¿ACELERA LA TRANSFORMACIÓN DIGITAL?
}

\author{
COVID-19: ACCELERATE DIGITAL TRANSFORMATION?
}

https://doi.org/10.52109/cyp2021115

Jimmy Velásquez ${ }^{1}$

${ }^{1}$ Investigador independiente

REGISTROS

Recibido el 15/08/2020

Aceptado el 30/09/2020

Publicado el 31/01/2021

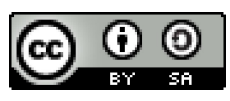

\section{PALABRAS CLAVE}

Transformación digital, pandemia,

covid-19,

ciudadanos digitales,

transformación digital.

\section{KEYWORDS}

Digital transformation, covid-19,

pandemic,

digital citizens,

digital transformation.

\section{RESUMEN}

El gobierno de cada país es promotor de emprendimientos y oportunidades para todos, que generen crecimiento económico igualitario en la sociedad, pero la realidad peruana y de muchos países es que el mayor porcentaje son micro, pequeñas y medianas empresas, las cuales son poco sostenibles en el tiempo y aún más sensibles a situaciones de coyuntura global como la pandemia del Covid-19 iniciada en el año 2,020. Desde antes de esta coyuntura creada por el Covid-19, en foros internacionales y nacionales bajo el marco de del Plan Nacional peruano de Competitividad y Productividad 2019-2030, señalan a las tecnologías de la información o tecnologías digitales como buena estrategia de innovación empresarial para promover un entorno competitivo menos desigual y con mayor productividad y por tanto más rentable para todas las empresas sin importar su tamaño.

Ahora, en tiempos del Covid-19, está más vigente la adopción de tecnologías digitales y de implementar en toda organización sin importar el tamaño, la tan necesaria Transformación Digital que permitirá a las empresas no solo agregar valor a sus clientes sino sobrevivir como negocio y ser más rentable. Esta coyuntura definitivamente está promoviendo de forma acelerada la adopción de tecnologías de mejor manera en todas las empresas y ampliando la experiencia digital en los clientes.

\section{ABSTRACT}

The government in each country is a promoter of entrepreneurship and make opportunities for all, which generate egalitarian economic growth in society, but in our reality in Peru and others countries exists high percentage of micro, small and medium sized companies exist, which are not very sustainable in time and even more sensitive to the global Covid-19 pandemic, that began in the year 2020. Since before this situation created by Covid-19, in international and national forums under the framework of The Peruvian National Competitiveness and Productivity Plan 20192030, they point to information technologies or digital technologies as a good business innovation strategy in order to promote a less unequal competitive environment with higher productivity and therefore more profitable for all companies regardless of their size. Now, in times of Covid-19 the adoption of digital technologies is more current and the implementation in all companies regardless of size is necessary, which will allow companies not only to add value to their customers but to survive as a business and be more profitable. This situation is definitely accelerating the adoption of technologies in a better way in all companies and expanding the digital customers experience. 


\section{INTRODUCCIÓN}

La tecnología digital, y más en la coyuntura ocasionada por la pandemia del Covid19, ha pasado ser el protagonista principal y necesario, en el quehacer de la sociedad desde las compras diarias, educación de hijos, trabajo, reuniones laborales y sociales, todo a través de Internet; estas prácticas que antes hubieran demorado años en conseguirlo de manera intensa llegaron para quedarse y seguir siendo protagonista y principal facilitador en la "nueva normalidad" post Covid-19.

Desde que apareció Internet nos sumergimos en una era en la cual la información se ha vuelto cada vez más necesaria para crear o sobrevivir en medio de los mercados cada vez más competitivos. Contar con información confiable y oportuna hace que cada vez se vuelva en el insumo necesario para toma de decisiones o establecer mecanismos para mantener a tus clientes o captar nuevos mercados. Si ahora estamos más informados y conectados, y podemos extender nuestro conocimiento más allá de lo que Internet nos permite, entonces deseamos que las empresas y organizaciones que me ofrecen algo sean más inteligentes e interconectadas, como las quiero y necesito.

Actualmente escuchamos diferentes conceptos y nuevas definiciones relacionadas al uso intensivo de la tecnología, siendo un concepto que engloba todo esto como la "4ta revolución", concepto mencionado durante el Foro Económico Mundial 2016 y por el fundador de este, Klaus Schwab. Después que el ser humano ya ha pasado por la mecanización, producción en masa, computación y automatización llegamos a la cuarta revolución, donde en base a la revolución digital la tecnología se integra a las sociedades e incluso a las funciones del cuerpo humano. Pero esta 4ta revolución, necesita un proceso de "Transformación Digital" en todas las organizaciones sin importar el tamaño y sector. El reto final de esta transformación es cómo mejoramos la experiencia del cliente (interno o externo a la organización) que está conectado por diversos dispositivos digitales y quiere ser atendido de la mejor manera.

Es bastante conocido y hemos leído por todos lados que el uso de las herramientas tecnológicas son un gran facilitador para cambios estructurales, de productividad e innovación. Pero esto no solo aplica para algunas empresas sino puede significar mucho para toda una economía si todas las empresas u organizaciones en general empiezan a aplicarla de alguna forma, incluido las microempresas y pequeñas empresas.

Actualmente los gobiernos y sociedades civiles que esta inmersos en transformaciones digitales día a día llevados por la moda o la necesidad de estar interconectados, están conscientes de iniciar una transformación digital que permita una gobernanza adecuada donde la sociedad civil deba ser parte de las decisiones que lleven a todos a un mayor desarrollo y bienestar. Visto de un contexto más amplio esto cubre desde un gobierno digitalizado como plataforma 
abierta que integra a los privados y el Estado para repensar procedimientos, procesos, servicios y capacidades.

\section{Pero ¿Qué es y qué no es Transformación Digital?}

Figura 1

Evolución del término "transformación digital" en las búsquedas latinoamericanas

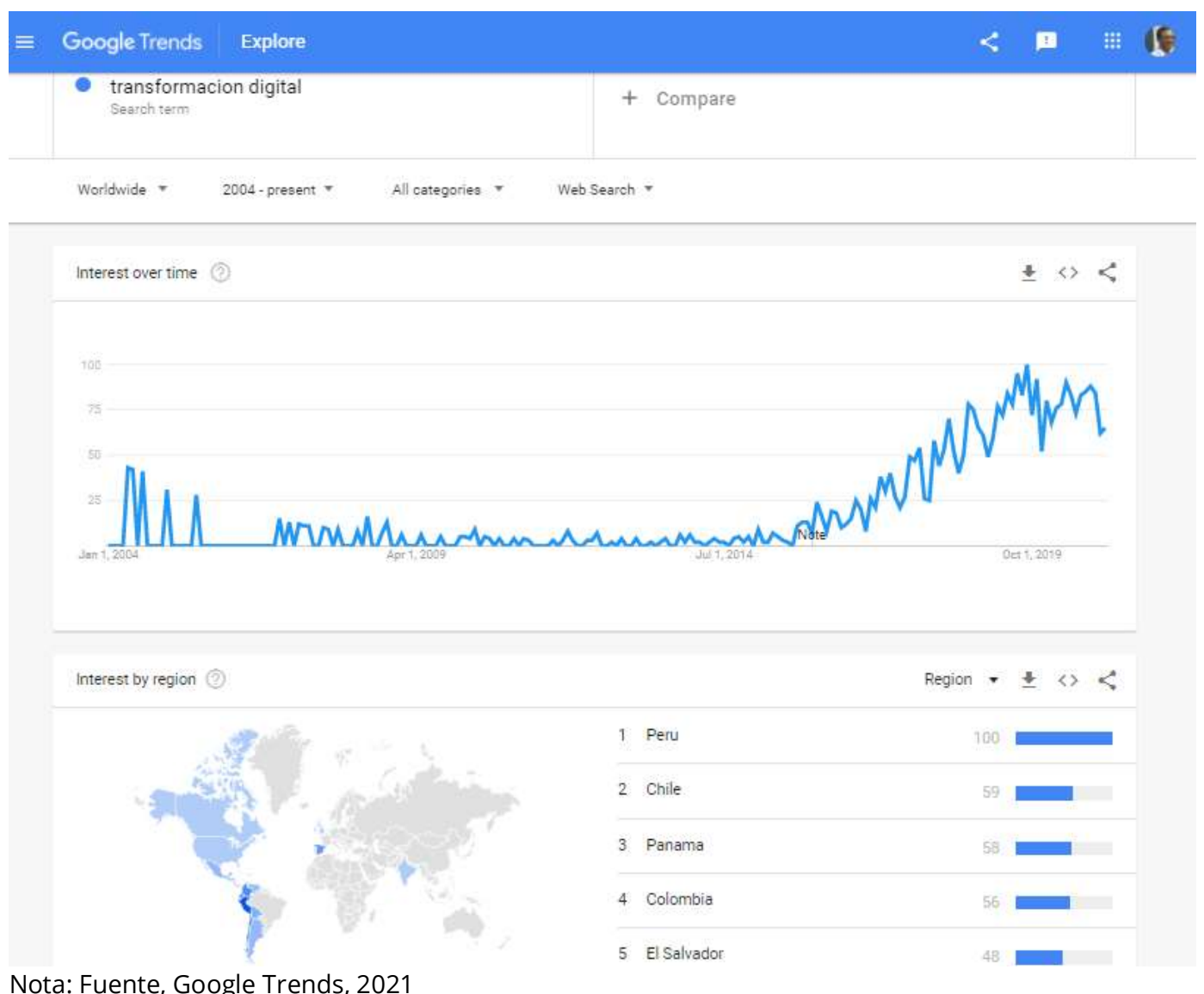

Nota: Fuente, Google Trends, 2021

La definición de transformación digital en la actualidad es un término más buscado que antes a través del buscador Google, por moda o por necesidad. Curiosamente el Perú lidera las búsquedas en países latinoamericanos, como lo muestra Google Trends.

Buscando una definición a Transformación Digital, en Internet nos podemos cruzar con diferentes enunciados y dada más por organizaciones privadas y con bibliografía en idioma inglés, entre las que podemos destacar las siguientes:

Según la enciclopedia libre Wikipedia "La transformación digital es el cambio asociado con la aplicación de tecnologías digitales en todos los aspectos de la sociedad humana. La transformación digital puede ser considerada como la tercera etapa o tercera fase de la adopción de las tecnologías digitales (la competencia digital $\rightarrow$ el uso digital $\rightarrow$ la transformación digital), junto con mejora de la capacidad 
de uso y de aplicación que se logra a través de la alfabetización digital". (Wikipedia, n.d.)

Según Gartner, la empresa de investigación y consultorías relacionados al sector de Tecnologías de Información y Comunicación define en su glosario: "La transformación digital puede referirse a cualquier cosa, desde la modernización de la TI (por ejemplo, la computación en la nube) hasta la optimización digital y la invención de nuevos modelos comerciales digitales. El término se usa ampliamente en organizaciones del sector público para referirse a iniciativas modestas como poner servicios en línea o modernización heredada. Por lo tanto, el término se parece más a "digitalización" que a "transformación empresarial digital". (Gartner Inc., 2020)

Según Salesforce, un proveedor líder en herramientas tecnológicas para Gestión de Relaciones con los clientes (CRM - Customer Relationship Management) y plataformas asociadas, define: “La Transformación Digital es el uso de la tecnología para mejorar radicalmente el rendimiento y el alcance de las empresas. Por un lado, la transformación digital es un imperativo que supone la creciente demanda de los consumidores de experiencias digitales sencillas e integradas a través de los distintos canales de servicio. Por otro lado, la transformación digital es ahora posible gracias a las diversas revoluciones tecnológicas que permiten a las empresas digitalizar todos los procesos de negocio". (Salesforce inc, 2017)

Según Hewlett Packard (HP), fabricante y comercializador líder en equipos de cómputo y software, define:" La transformación digital es un proceso que integra la tecnología digital en todos los aspectos del negocio y que requiere de cambios fundamentales en el ámbito de la tecnología, la cultura, las operaciones y la entrega de valor. Para aprovechar mejor las tecnologías emergentes y su rápida expansión en las actividades humanas, las empresas deben reinventarse y transformar radicalmente todos sus procesos y modelos." (Hewlett Packard, 2020)

Según Citrix, empresa líder en tecnologías de virtualización de servidores, conexión en red e informática en la nube, define "La transformación digital es la adopción estratégica de tecnologías digitales. Se utiliza para mejorar los procesos y la productividad, entregar mejores experiencias a los clientes y empleados, gestionar el riesgo empresarial y controlar los costos. La transformación digital representa innumerables herramientas, soluciones y procesos. Una estrategia efectiva es una personalizada para cada organización única." (Citrix Systems Inc, 2020)

Todas estas definiciones coinciden en 3 conceptos importantes: Cambios, Tecnología Digital y entrega de valor al cliente. Cambios en la cultura organizacional, procesos de negocio y mejora de capacidades de las personas; cambios soportados sobre Tecnología Digital como medio o principal facilitador y todo ello orientado para agregar valor en los productos o servicios entregados al cliente, haciendo a la organización más efectiva y rentable. Suena bastante coherente y ahora más que 
nunca el reto es aplicarlo a la realidad de cada organización comercial o no sin importar su tamaño.

Hoy en día, la mayoría de las personas ya han utilizado algún equipo o solución basada en tecnologías digitales, y más ahora durante la pandemia del Covid-19 desde compras en línea, entretenimiento o realización de alguna gestión administrativa. Mucha de esta tecnología digital de uso cotidiano está siendo usadas en la sociedad humana aun sin comprender su complejidad, pero motivados por necesidad diaria.

Muchas de estas tecnologías digitales están bastante maduras y son muy conocidas actualmente, pero otras están en proceso de maduración y uso general, entre las que podemos destacar:

-Inteligencia Artificial que trata de simular el razonamiento automático del cerebro humano especializado en diversos temas. Puede ser la tecnología más disruptiva y de transformación y revolución en todos los sectores.

- Tecnología IOT (Internet of Things o Internet de las cosas) para que todos los dispositivos electrónicos que usamos o usaremos estén interconectados y sean de alguna forma autónomos.

- El Bigdata para administrar alto volumen de datos, generados cada vez más rápidos y por muchos canales y más adelante datos generados también por la IOT.

- Los SmartCities (Ciudades Inteligentes) donde en base el IOT, los componentes de la ciudad serán auto gestionados desde centrales inteligentes.

- BlockChain como tecnología o protocolo de confianza que ofrece mayor seguridad en las transacciones. Actualmente usado en el comercio de criptomonedas como el Bitcoin, Ethereum y muchas otras Altcoins.

- Impresión en 3 dimensiones, actualmente usado para creación de piezas en muchos sectores, incluido en prótesis para el ser humano.

- Nanotecnología para la manipulación de la materia a escala manométrica (igual a 1 milímetro entre un millón de partes) usando tecnología y la biotecnología.

- Computación cuántica basado en el uso de cubits (unidades distintas al bit y que puede superponer a la vez sus 2 valores) y con capacidad de procesamiento según Google quien ya ha creado una (no es la única empresa) necesitaría 200 segundos para resolver un problema que le tomaría a la supercomputadora más rápida del mundo 10,000 años resolver

Entonces la interrogante es ¿La Transformación Digital está relacionada solo al uso de estas tecnologías?, la respuesta es no. Es necesario comprender que, de acuerdo a los procesos de negocio de cada organización se vayan adoptando parte de estas tecnologías u otras complementarias o sustitutorias, pero que les agreguen valor a sus clientes sobre todo y no sea una implementación guiada solo por la moda. Esto lo logramos primero elevando las capacidades digitales de las personas que trabajan en una organización, para que desde el conocimiento adquirido utilicen las herramientas existentes y con un conocimiento y uso bien gestionado se fomente 
la innovación para finalmente agregarle valor al cliente. Todo esto en conjunto permitirán lograr la transformación digital de forma sostenible en la organización. En esta parte podemos resumir, que la implementación de herramientas digitales no debiera ser el inicio de este proceso, por sí solo no es transformación digital, sino debería ser primero la elevación de capacidades a las personas que lo usarán, pero con enfoque de innovación para mejorar o cambiar los procesos del negocio y finalmente agregarle valor al producto o servicio entregado al cliente.

\section{MATERIALES Y MÉTODOS}

Para la elaboración del presente artículo se ha requerido, examinar a través del Buscador Google: libros, artículos, foros, websites corporativos, entre varias fuentes oficiales o comerciales; primero las diferentes definiciones que se han ido desarrollando en los últimos años, donde se percibe mayor interés para que la tecnología se convierta en un aliado estratégico de toda organización, sea empresa comercial o no. Una vez consensuado el significado a través de las definiciones dadas por empresas importantes e íconos según su rubro, se ha extraído 3 conceptos importantes a tomar en cuenta en la aplicación a la realidad de cualquier organización: Cambios, Tecnología Digital y entrega de valor al cliente.

Después de consensuar una definición, se ha revisado el contexto internacional, tanto de las experiencias de las empresas y planificación de sus CEO (Chief executive officers); muchas de estas empresas basadas en tecnología como parte central del negocio, han rentabilizado más aun en esta coyuntura generada por la pandemia Covid-19.

Finalmente se hace una revisión del contexto nacional, cómo venía desarrollándose la Transformación Digital en el Perú tras la llegada de la pandemia del Covid-19, tomando de referencia principalmente el estudio realizado en el 2019 por Everis Perú llamado "Transformación Digital en el Perú".

\section{RESULTADOS}

Las pequeñas empresas mayormente empiezan la transformación digital mediante la adquisición de aplicaciones digitales y logran un nivel básico de digitalización, mientras que las empresas medianas y grandes logran un mayor nivel de digitalización porque empiezan por cambios en la cultura organizacional soportado después por herramientas tecnológicas.

La transformación digital es un proceso impostergable dentro de las organizaciones sin importar su tamaño. Procesos que involucran cambios en la forma de pensary realizar la oferta de servicios o productos que le agreguen valor al cliente, dichos procesos deben ser potenciados con el uso de herramientas tecnológicas de acuerdo con la realidad de cada organización. 


\section{DISCUSIÓN}

\section{El contexto de transformación Digital}

Es indiscutible el rol que tiene la adopción de las tecnologías digitales y como lo manifiestan $66 \%$ de CEOs según datos publicados por IDC (International Data Corporation) donde indican que los planes de negocios de las empresas tienen como pieza clave la transformación digital.

Por poner otro ejemplo, un informe de Price Waterhouse Coopers, siendo una de las principales firmas consultoría y auditoría de empresas, indica que de 867 directores financieros (Chief Financial Officer - CFO) entrevistados en 24 países sólo el $16 \%$ de ellos piensa rebajar el presupuesto en transformación digital tras la pandemia COVID-19. Todos están conscientes que el control de costes como estrategia de estabilización es necesario, pero también coinciden, que la inversión en transformación digital es prioritario e irremplazable en la estrategia de sus organizaciones para lograr estabilizarse.

El uso de tecnologías basado en Internet, no es novedad que crece cada año, pero ¿Las organizaciones son conscientes de lo que está pasando y donde están más tiempo las personas?, los números son sorprendentes, incluso para los que estamos inmersos en el desarrollo de tecnologías y sistemas de información; sabias que cada 60 segundos se realizan más de 4.1 millones de consultas a Google, se envían 59 millones de mensajes con Whatsapp, se ven 764,000 horas de videos en NetfLix, se visitan 4.7 millones de videos en Youtube, 1.1 millones de ventas online y muchos otros ejemplos.

Y estos números siguen incrementado cada vez más, debido a la pandemia del COVID-19, que nos ha tocado vivir a todas las generaciones actuales, por solo dar un ejemplo y como lo indica en el estudio de cómo ocupan su tiempo las personas y el uso de medios digitales "Coranavirus Research - Media Consumption and Sport" (Global Web Index, 2020) y diferenciando a las generaciones nos indica a manera de ejemplo que el $42 \%$ de baby boomers(nacidos a mitad del siglo 20) consumen más televisión y 1 de cada 5 a través de streeming (Televisión online), que más de la mitad de la Generación Z(nacidos en los años 1997 en adelante) consume videos por Youtube o Tik Tok y respecto a la generación Millenial (nacidos en los años 1980) el $30 \%$ de consumen streamming y el $20 \%$ podcasts.

El mundo y la economía digital que muchos estaban recién entendiendo, definitivamente después de esta coyuntura iniciada por el COVID-19, se ha acelerado. Como ya en algunos artículos lo definen en "60 días de confinamiento por el COVID-19 han acelerado 6 años la digitalización del mundo" y como lo explica Nacho de Pinedo, CEO de ISDI, quien hace una radiografía del profesional post Covid-19, que deberá ser capaz de adaptarse para acompañar a las empresas en sus procesos de digitalización (El Confidencial, 2020).

En este contexto la pregunta es y cae por su propio peso, ¿La transformación digital cómo interviene en todo este contexto acelerado por la pandemia Covid-19?, si 
partimos de la premisa que las empresas y organizaciones en general nacen para crear riqueza o beneficios a través de la oferta de productos o servicios y estos se brindan para satisfacer las necesidades o deseos de las personas; entonces la respuesta también cae por su propio peso, es necesario estar más cerca a las personas y a través de los medios que ahora conocen más y utilizan más, y estos son los medios digitales.

Desde hace algunos años se viene escuchando sobre la transformación digital en las organizaciones, pero para ser sinceros, siempre creemos y porque puede parecer una secuencia lógica que estos cambios son exclusividad o al menos empiezan con las grandes organizaciones que ya cuentan con áreas de tecnología que vienen digitalizando sus procesos. Esto tiene bastante correlación con su definición y como una etapa final dentro de la adopción de tecnologías digitales, pero no es y no debería ser solo exclusividad de estas organizaciones. Para completar esta adopción de tecnologías digitales en una organización sin importar su tamaño se tiene que reconstruir dinámicas de procesos del negocio donde primero es necesario que los agentes del cambio (administradores y trabajadores) cambien sus paradigmas y se alineen en una sola estrategia de adopción de estas tecnologías. Esta estrategia involucrará que eleven sus capacidades tecnológicas para gestionar la información que se generan por diversas fuentes, formatos y canales, para que aprendan a gestionar el conocimiento adquirido, para que se fomente el autoaprendizaje y puedan evolucionar al ritmo de los cambios tecnológicos, para realizar trabajo colaborativo que mejore las sinergias y eficiencia en el trabajo, para diseñar experiencias participativas y multicanal con los clientes(páginas web, redes sociales, emails, chat, llamadas, foros entre otras).

Un ejemplo claro y actual de negocio basado en tecnologías digitales son las startups que desde que nacieron muchas de ellas adoptaron y centraron sus procesos de negocio basado en tecnología, sumado a la innovación que proponen, son más rápidas para desarrollar, probar, operar y escalan más rápido que otras empresas que quizás en el mismo rubro llevan más años de operación. Muchas de estos startups son las llamadas empresas Unicornio, que han conseguido una valoración de más de 1.000 millones de dólares en aproximadamente 3 años, antes de salir a la bolsa de valores. Para mencionar algunas y que son bastante conocidas estan: ByteDance (creador TikTok), Wish (e-commerce basado en), Numbank(fintech), DoorDash (Delivery comida), Epic Games (Creador de Fornite), Airbnb (Hospedaje turístico), Mercado Libre(e-commerce), Uber (transporte urbano), entre muchas otras.

Estos ejemplos ahora pueden sonar a grandes inversiones desde el inicio, pero no todas nacieron así, lógicamente empieza con una idea bien planteada y luego los inversores van llegando si se sabe buscar y sustentar.

Ya no es posible imaginar una organización moderna y con visión de futuro que no cuente con un plan de negocios basado en la adopción de tecnología digital. Esto no significa que durante el proceso no exista "el fracaso", término aún vetado en la 
idiosincrasia actual de las organizaciones privadas y estatales, pero por el contrario todo cambio importante puede conllevar a varios fracasos antes del éxito planificado. La innovación tiene como matiz necesario el fracaso y es necesario que los lideres inteligentes, equipos y emprendedores pongan el fracaso a trabajar a nuestro favor, como lo expone John Danner y Mark Coopersmith en su libro "The Other ' $F$ ' Word. How Smart leader, Teams and Entrepreneurs put Failure to Work" (Danner \& Coopersmith, 2015).

La innovación tiene como factor clave al proceso de transformación digital, y más ahora que en la actualidad existen metodologías ágiles que ayudan a ser disruptivo y en base a innovación con tecnología fomentar cambios importantes y ágiles en la organización. Incluso a desechar una opción en etapas tempranas que no involucren muchos recursos invertidos, justamente la agilidad permite en cortos tiempos desarrollar, probar, corregir o desechar para iniciar un nuevo camino. Tan vigente aun la frase de uno de los más famosos inventores de América, Tomás Edison decía" No he fallado, he encontrado exitosamente 10,000 maneras que no funcionan".

Una prueba importante la estamos pasando y viviendo actualmente originada por la pandemia COVID-19, donde la adopción de tecnologías digitales y cambios en sus procesos de negocio han cambiado tan rápido y en tiempo récord para muchos. Desde empresas unipersonales o negocios ambulantes hasta las empresas medianas o grandes que ya lo venían haciendo.

Por lo expuesto, la transformación digital debe ser considerado el proceso general dentro de la organización sin importar su tamaño, donde se completa la adopción de tecnologías digitales como parte integral de los procesos del negocio para interactuar con los clientes y entregarles un mayor valor en sus productos o servicios.

\section{Transformación Digital en el Perú}

En el CADE 2019 realizado en Perú, se concluyó que la ruta de la competitividad es digital y basada en la gobernanza de los datos. En el CADE Digital 2020, evidencia que la transformación digital puede y debe ser el motor para la reactivación económica y social del país.

El Estado Peruano consciente de estos retos impuestos por una economía globalizada y el uso creciente de las tecnologías digitales, desde la creación de la Secretaría de Gobierno Digital en el 2017 como ente rector del Sistema de Transformación Digital y Política Digital en el Perú. y en el marco de su Política Nacional de Competitividad y Productividad suscrita en diciembre 2018, emitió en Julio 2019 su Plan Nacional de Competitividad y Productividad 2019-2030 haciendo mención en sus objetivos prioritarios el "Desarrollo de Capacidades para la Innovación, Adopción y Transferencias de Mejoras Tecnológicas" donde se resalta que la innovación empresarial no puede dejar de lado la trasformación digital. 
Todos estos objetivos planteados en el Plan Nacional y de la mano de la transformación digital ayudará a que el país pueda lograr una mayor diversificación en su economía, ayudará afrontar los retos de baja productividad en sectores críticos de su economía y promoverá un desarrollo igualitario entre sus regiones. Como lo indica textualmente uno de los imperativos del mencionado Plan Nacional es reducir la informalidad de las micro y pequeñas empresas para consolidar un entorno empresarial más competitivo, racionalizar los trámites y procurar un marco regulatorio razonable y eficiente; que es vital, como lo ha establecido la literatura económica, para estimular el emprendimiento y la productividad en los países.

En enero del 2020 con un decreto de urgencia se iniciaba una nueva etapa, con la creación del "Sistema Nacional de Transformación Digital" (El Peruano, 2020) para impulsar la modernización de la Gestión Pública en base a la integración de plataformas digitales entre los diversos sectores económicos y sociales, desde el Estado a las empresas privadas grandes, medianas, pequeñas y microempresas. Una vez creado el Sistema Nacional se necesita la Política y Estrategia Nacional de Transformación Digital, tomando mayor relevancia con la declaración mundial la pandemia del Covid-19. Siendo esta época cuando más importante es tener las reglas claras y el apoyo desde el Estado con lineamientos, gestión pública optimizada y sobre todo la inversión que siempre se necesitó para Investigación, Desarrollo e Innovación (I+D+i); pero bueno ya no es tiempo de lamentarse de lo que no se hizo sino de la oportunidad de hacerlo ahora, mantenerlo y mejorarlo post Covid-19. Todo debe acelerarse para que muchas empresas sobrevivan y se reinventen.

Luego de revisar el informe del estudio subnacional elaborado por primera vez en Perú “Doing Business en el Perú 2020" publicado el 04 de junio del 2020 (Banco Internacional para la Reconstrucción y el Desarrollo / Banco Mundial, 2020), basado en datos de los años previos concluye que en el Perú, tomando como muestra 12 principales ciudades, comparado con otras 189 economías y en base a 4 indicadores que determinan la facilidad con la que se hace negocios, ubica al Perú como una de las más complicadas para la apertura de una empresa y en un nivel intermedio para construcción, registro de propiedad y cumplimiento de contratos. Pero lo que más resalta es que sin importar el tamaño de la región o ciudad algunos indicadores fueron mejores fuera de la capital, como en Ancash donde incluso hay escaso acceso a medios digitales. A pesar de que en algunas ciudades se obtienen buenos resultados con el apoyo de soluciones digitales, aun la brecha digital es muy grande por atender en el Perú.

Según el mismo informe “Doing Business in Peru 2020", con relación a la apertura de un negocio se resume lo siguiente:

- La mayoría de los emprendedores en el Perú sigue prefiriendo realizar los trámites para abrir una empresa de manera presencial, a pesar de los esfuerzos de la Sunarp por ofrecer los servicios en línea. La excepción a la regla es Lima, que lidera las inscripciones en línea 
- Abrir una empresa en el Perú tarda alrededor de 17 días más que en el promedio de Latinoamérica, aunque es un $70 \%$ menos costoso. Comparado con el promedio de los países OCDE de altos ingresos, tarda casi un mes y medio más, requiere 3 trámites extra y cuesta casi 4 veces más

- La obtención de la licencia de funcionamiento en el Perú es el proceso más largo y costoso para abrir una empresa; llegando a significar el uso del 73\% del tiempo de los trámites y alrededor de un $40 \%$ del costo total. Realizar este trámite es más sencillo en Lima y en Arequipa.

- Subsisten retos para la apertura de una empresa en el Perú como el uso progresivo de los sistemas en línea para el registro y conseguir que el proceso de obtención de la licencia de funcionamiento sea unificado y ágil.

Si esta es la realidad de nuestra economía, y eso explica la mayor informalidad que se aprecia, la pregunta es ¿Cómo encontró a las empresas del Perú esta pandemia del COVID-19?, la realidad es que pocas empresas estaban en proceso de implementación de su transformación digital y la mayoría sin entenderla y peor aún sin conocer de qué se trata. Los ejemplos en estos temas de avances en transformación digital son liderados en su mayoría por empresas grandes o medianas.

De acuerdo con el libro "Transformación Digital en el Perú" elaborado por Everis Perú SAC, en el Perú de la mano de proveedores de tecnología, consultoras o casas matrices, algunas empresas se embarcaron en procesos de transformación, pero recién en el 2017, el tema ingresó a las agendas corporativas, aunque con cierta confusión y ruido. Como se describe en este libro, la transformación digital como práctica e inicios en el Perú empieza en el 2017 y solo con algunas empresas básicamente del sector Financiero y Telecomunicaciones. Algunos reconocibles esfuerzos también se dan en empresas con operaciones fuera de la ciudad, como es en el sector minero con la implementación de centros de control remoto que centralizan las operaciones de monitoreo (Saldarriaga, 2020). 
Figura 2

Nivel de digitalización y elemento para iniciar la transformación digital según tamaño de empresa

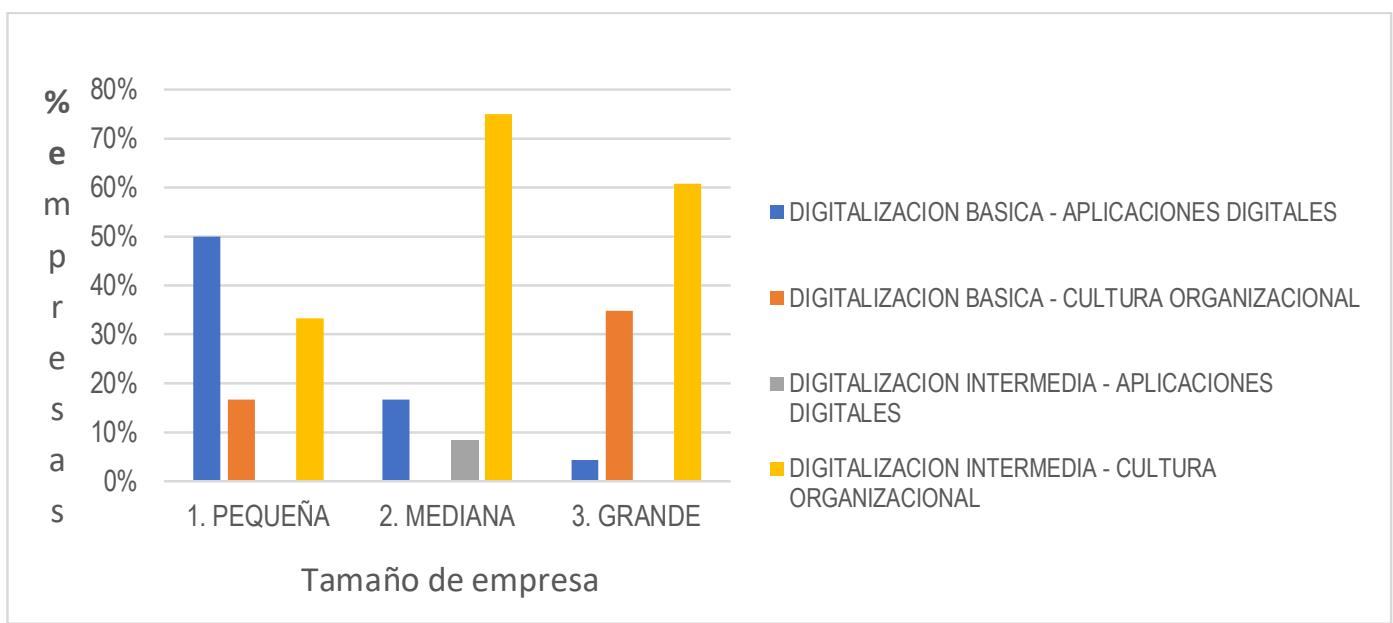

Nota: Elaboración propia basada en el libro "Transformación Digital en el Perú" elaborado por Everis Perú S.A.C.

En la figura 02, se muestra que más del 50\% de empresas medianas ( $>250$ y $<=1000$ trabajadores) y grandes ( $>1000$ trabajadores) alcanzaron un mayor nivel de digitalización cuando iniciaron su transformación digital a partir de los cambios en su cultura organizacional, mientras que el 30\% de pequeñas empresas $(<=250$ trabajadores) lo lograron bajo el mismo enfoque. El 50\% de las pequeñas empresas iniciaron su transformación digital con herramientas informáticas o aplicaciones digitales y han alcanzado una digitalización básica.

Según lo mostrado en la gráfica de la figura 02, las pequeñas empresas mayormente empiezan la transformación digital por aplicaciones digitales y logran un nivel básico de digitalización, mientras que las empresas medianas y grandes logran un mayor nivel de digitalización cuando empiezan por cambios en la cultura organizacional.

“Más que buscar ser los primeros en transformación digital, se trata de un tema de sobrevivencia. Si este proceso no lo hacemos con decisión, no vamos a estar a la altura de nuestros clientes financieros del siglo XXI." (Llosa, 2019)

"A veces uno pone el coche delante de los caballos. De nada sirve ser digital si las soluciones no son relevantes, lo que para nosotros significa liderar la satisfacción del cliente. Uno de los caminos para lograr ese objetivo es la transformación digital." (Ucelli, 2019)

“La centralización del cliente y la innovación son los ejes fundamentales que sigue toda la organización para alcanzar la transformación digital en el Perú." (Revuelta, 2019) 
"Cuando se habla de transformación digital, muchos piensan en herramientas. Cuando la empresa inició este proceso, nos dimos cuenta de que, si nuestra cultura corporativa no cambiaba, las inversiones que estábamos haciendo no iban a agregar valor al negocio." (Ghio, 2019)

“Muchas veces, los jugadores tradicionales piensan en usar el celular y las nuevas tecnologías como un canal de ventas o para simplificar los procesos existentes, pero no se toman el tiempo de empezar de cero y recrear productos, servicios o negocios." (Roca Zegarra, 2019)

“El mercado siempre será el gran termómetro. Creo que todas las organizaciones privadas nos hemos dado cuenta de que, si no nos transformamos, desapareceremos." (Ichazo Bardales, 2019)

“La transformación digital para brindar a nuestros clientes actuales una experiencia diferente y mejor, sobre todo en los procesos internos, donde nuestro negocio ha sido muy manual." (Correa, 2019)

“Tenemos que ir creando cultura. El uso de herramientas digitales es una forma espectacular de mejorar la experiencia de nuestros clientes y ya estamos viendo resultados positivos" (Ríos Sarmiento, 2019)

“Gobierno, empresas y ciudadanos tienen que ser cada vez más digitales, no solo como consumidores, sino también como productores de tecnología. Los países que sepan aprovechar los beneficios de la economía digital serán los más exitosos" (Cortez, 2019)

"La transformación digital se tiene que entender como el aprovechamiento de la tecnología para atender las necesidades de las personas y brindarles mayores oportunidades" (Belmont, 2019)

“El objetivo central de la transformación digital no es reducir costos. Se busca que la empresa siga operando exitosamente en el futuro, con consumidores satisfechos, que prefieran una marca. El cliente final es el que cuenta para definir el nuevo modelo y la estrategia de negocios, en función a segmentos de mercado y no de productos" (Graham, 2019)

Es muy importante saber cómo piensan los principales gestores, CEOs y directores de cada empresa u organización del Perú, y en su mayoría coinciden que la transformación digital empieza con el cambio de la cultura organizacional y centrada en el cliente, para después potenciarlo con metodologías y herramientas tecnológicas.

Lo que siempre esperábamos y como lo enfoca las buenas prácticas del marco de trabajo para el Gobierno y la gestión de la información y tecnología de cualquier organización (COBIT - Control Objectives for Information and Related Technologies), 
que las metas corporativas estén bien alineadas a las metas de Tecnologías de Información y por tanto la convierten no solo en un área de soporte sino en un aliado estratégico.

\section{¿Y las Pequeñas y Microempresas?}

Las Micros y pequeñas empresas son esenciales en la economía del país, emplean a más de la mitad de la población económicamente activa como lo indica en su publicación de "Producción y empleo informal en el Perú. Cuenta Satélite de la Economía Informal 2007-2017" publicado por el INEI en 2018. Sin embargo, el 80\% generan empleos informales. Basada en esta informalidad la pregunta es ¿Cuántas de estas Pequeñas y Microempresas podrán implementar la Transformación Digital de alguna forma y que les permita continuar?

Pareciera que estos temas solo aplican a las grandes o medianas empresas, pero realmente cuando la necesidad apremia, el ingenio sobresale y equilibra en varios casos las deficiencias, pero tiene que ser sostenible en el tiempo para que realmente se obtenga un beneficio real.

Un ejemplo en este tipo de empresas para iniciar una implementación de Transformación Digital desde y con el Estado podemos mencionar el ejemplo de los vendedores de emoliente o emolienteros (venta en la calle en puestos móviles, de bebidas calientes en base a hierbas y productos naturales) y para cumplir protocolos de protección contra COVID-19, ahora cobran a través de tarjetas de crédito o débito mediante POS móviles, todo esto con apoyo y soporte del Ministerio de Producción, como lo describe el artículo web “Emolienteros cobrarán con tarjetas para prevenir contagios de coronavirus" (UCI.pe |R. Andrés, 2020).

En este ejemplo cambió un proceso dentro del negocio, el realizar el cobro por un medio digital de uso popular (POS móvil y más adelante seguramente mediante chip del celular) y de hecho también cambiará el proceso para realizar el cuadre de ingresos y empezarán a publicitar sus productos de diversas formas y por diferentes medios como las redes sociales (Facebook, WhatsApp, entre muchos otros), es decir, poco a poco será transversal a los principales procesos del negocio. La idiosincrasia cambia primero y luego se potencia con el uso de las tecnologías digitales.

Según definición esta adopción de tecnologías digitales sería una forma de inicio de transformación digital a menor escala, donde se cumplen los tres conceptos: cambio en un proceso, uso de tecnología digital y agrega valor al cliente ofreciéndole mayor seguridad y comodidad en su pago.

\section{Entonces ¿Qué hacer?}

Lo primero, para cualquier organización sin importar el tamaño, es no abrumarse con todas las tecnologías a disposición porque empezar a usarlas sin un objetivo claro no tiene sentido. Primero es cambiar el "mindset" o forma de pensar de quien las dirige y estar incluso dispuesto al cambio que vendrá por varios lados: modelo de negocio, forma de generar ingresos, cambios la forma como se contacta con los 
clientes, cantidad de transacciones, entre muchos otros. Todo esto es parte de una investigación interna dentro de la organización, pero para repensar y encontrar lo que realmente requieren sus clientes de acuerdo con su comportamiento, preferencias, necesidades y tendencias.

En una pequeña empresa, no necesariamente pasa por implementar una herramienta tecnológica costosa. El primer paso es, él mismo, cambiar la forma de pensar e identificar lo más básico que puedan necesitar sus clientes, por ejemplo, un negocio de venta de comida o ropa y todos los productos o servicios que se nos ocurra comercializar, ahora por las necesidades del confinamiento han empezado a publicitar por Internet y redes sociales y utilizan el delivery para la entrega. Si a esto le sumamos que también se empiece a recolectar los emails de contacto o teléfonos de sus clientes ya podrán ir generando una base de datos inicial que pronto se podrá convertir en el inicio de nuevas campañas de fidelización y atracción.

Esta coyuntura y época de confinamiento ha servido a muchos negocios pequeños, sobre todo, a reinventarse, por ejemplo, de vender comida a vender suministros para prepararlas vía internet, de venta de ropa a venta de protectores y mascarillas vía internet y redes sociales, de shows y clases presenciales a transmisiones a distancia vía Zoom y muchos más casos. Todos estos ejemplos sin dudarlo ya dieron el primer paso de un gran cambio, motivados ahora por necesidad de sobrevivencia del negocio y más adelante será el inicio de una transformación digital ¿por qué no? ¿qué lo impediría si el primer gran cambio ya lo dieron?

\section{Transformación digital en tiempos del COVID-19}

Desde varios meses antes del inicio oficial de la pandemia del COVID (marzo 2020), ya se hablaba en casi todas las conversaciones en el ámbito tecnológico, de la transformación digital en las empresas. Como todo cambio de fondo, normalmente empieza primero en las empresas grandes y en orden descendente de acuerdo con el tamaño de la empresa, pero con el inicio de la pandemia todo se ha acelerado sin ningún orden en específico. Para las poblaciones que aún se resistían a usar medios digitales para cualquier trámite ya se hizo una necesidad y rompieron la barrera de la desconfianza en medios digitales, lo cual trae más posibilidades para las empresas en aprovechar el contacto con más personas digitalizadas, también otra oportunidad para muchas empresas en potenciar e incluso sobrevivir en el mercado.

Esta pandemia no solo cambiará la manera de ver y sentir las cosas que antes eran "normales" ahora esta será una "nueva normalidad" donde los procesos se reinventaron o potenciaron:

- Teletrabajo marcará un nuevo hito en las empresas que antes no creían en el trabajo a distancia, se está demostrando que muchos trabajos se pueden realizar sin problemas a distancia y de forma colaborativa.

- Los negocios pequeños y de venta directa como era de los alimentos, ahora es más común hacerlos usando las redes sociales y utilizando el delivery para la 
entrega. Ya no es exclusividad de empresas grandes, ahora desde la bodega de la esquina o vendedor del mercado te lo pueden hacer llegar y cobrar directamente o incluso usando medios de pago móviles, esto último ayudarán en la bancarización.

- Teleeducación, desde cursos independientes, nidos, colegios, Institutos y Universidades han volcado la enseñanza a través de videoconferencia vía Internet.

- Telesalud, por ahora para atención y diagnóstico por referencias de síntomas o visualización a distancia mediante celulares $u$ otros dispositivos con conectividad a Internet.

Según datos de la Encuesta Nacional de Hogares elaborado por el Instituto Nacional de Estadística e Informática del Perú donde se indica que el $40.1 \%$ de hogares tiene acceso a Internet (la mayor brecha es en zona rural con un 5.9\%), más del 93\% de hogares cuentan con al menos una persona con celular. Esto demuestra que hay más personas conectadas cada vez y más en zonas urbanas. Con toda esta población conectada y con la experiencia digital ganada en esta época que ha hecho crear más confianza a las empresas y a los clientes, abre un abanico de nuevas posibilidades para todos, lo que antes solo era casi exclusividad de las empresas grandes.

Dentro de todo el impacto negativo de la pandemia, lo positivo que podemos rescatar es esta experiencia digital ganada por las personas (rumbo a convertirse en ciudadanos digitales) en su quehacer diario y cambios que están realizando las empresas sin importar el tamaño para atenderlos, aunque aún hay una brecha digital de conectividad sobre todo en zonas rurales, para lograr una transformación a nivel país; pero es el inicio del cambio.

\section{CONCLUSIONES}

En resumen, la transformación digital no es solo un proceso de cambio importante sino impostergable, dentro de las organizaciones sin importar su tamaño, por el cual se debe atravesar de manera consciente y con toda la organización involucrada; potenciada con el uso de herramientas tecnológicas de acuerdo con la realidad de cada organización y que por sobre todo les ayuden a acercarse y conocer cada vez más a su público objetivo.

Todo ello conlleva a un reto muy importante para toda organización, que tomara tiempo pero que una vez implementado sentará la bases para un crecimiento ordenado rentable y que entregue el máximo valor al cliente.

\section{RECOMENDACIONES}

Toda organización si quiere sobrevivir como negocio y ser rentable en el tiempo debe iniciar su proceso de transformación digital, de acuerdo con su realidad. Siempre teniendo en cuenta en agregar valor al cliente desde cambios en la forma 
de pensar en la gestión como en la operación del negocio y potenciado con el uso de aplicaciones digitales o herramientas tecnológicas al alcance y estrictamente necesarias según el negocio.

\section{REFERENCIAS}

Banco Internacional para la Reconstrucción y el Desarrollo / Banco Mundial. (2020). Doing Business in Perú 2020.

Belmont, Y. (2019). Cultura de innovación en Everis Perú (Ed.), Transformación Digital en el Perú. Pentagraf.

Citrix Systems Inc. (16 de 08 de 2020). ¿Qué es la transformación digital? Obtenido de Transformación Digital: https://www.citrix.com/es-es/glossary/what-isdigital-transformation.html

Correa, A. (2019). Aprendizaje constante en Everis Perú (Ed.), Transformación Digital en el Perú. Pentagraf.

Cortez, P. (2019). Transformación basada en el cliente en Everis Perú (Ed.), Transformación Digital en el Perú. Pentagraf.

Danner, J., \& Coopersmith, M. (2015). The Other "F" word. How Smart leader, Teams and Entrepreneurs put Failure to Work. New Jersey - United States: John Wiley \& Sons, Inc.

Decreto de urgencia 006-2020 de 2020. Decreto de urgencia que crea el Sistema de Nacional de Transformación Digital. 08 de enero de 2020. Diario Oficial 3.

El Confidencial. (11 de 06 de 2020). Los 60 días de confinamiento han acelerado seis años la digitalización del mundo. Obtenido de El Confidencial: https://www.elconfidencial.com/tecnologia/2020-06-11/transformaciondigital-isdibra_2622219/?fbclid=IwAR2RBeQ7uFkkZ7NFvVdbdmXcl3Ud4B3aNrk6kljnT3 9w_MM7s819xcdX5s

Ferrand Aspillaga, L. (2019). Transformación transversal en Everis Perú (Ed.), Transformación Digital en el Perú. Pentagraf.

Ferrini, A. (2019). Adaptación cultural en Everis Perú (Ed.), Transformación Digital en el Perú. Pentagraf.

Gagliuffi, I. (2019). Alto impacto en Everis Perú (Ed.), Transformación Digital en el Perú. Pentagraf.

Garcia de Fabbri, M. (2019). Transformación sobre ruedas en Everis Perú (Ed.), Transformación Digital en el Perú. Pentagraf.

Gartner Inc. (16 de 08 de 2020). Information Technology - Gartner Glossary. Obtenido de https://www.gartner.com/en/informationtechnology/glossary/digital-transformation

Ghio, A. (2019). El futuro es hoy en Everis Perú (Ed.), Transformación Digital en el Perú. Pentagraf.

Global Web Index. (abril de 2020). Global Web Index. Obtenido de Global Web Index: https://www.globalwebindex.com

Graham, C. R. (2019). El cliente es el eje en Everis Perú (Ed.), Transformación Digital en el Perú. Pentagraf. 
Hewlett Packard. (16 de 08 de 2020). ¿Qué es la Transformación digital? Obtenido de https://www.hpe.com/es/es/what-is/digital-transformation.html

Llosa, E. T. (2019). Goles digitales en Everis Perú (Ed.), Transformación Digital en el Perú. Pentagraf.

Ichazo Bardales, J. (2019). Inclusión con foco digital en Everis Perú (Ed.), Transformación Digital en el Perú. Pentagraf.

Mannheim, A. (2019). Información para el mundo em Everis Perú (ed.), Transformación Digital en el Perú. Pentagraf.

Price Waterhouse Cooper. (22 de 05 de 2020). ¿Qué están haciendo los CFOs en la fase de estabilización de la crisis? Obtenido de https://ideas.pwc.es/archivos/20200522/que-estan-haciendo-los-cfos-en-lafase-de-estabilizacion-de-la-crisis/

Ríos Sarmiento, F. (2019). Creando bienestar en Everis Perú (Ed.), Transformación Digital en el Perú. Pentagraf.

Revuelta, J. M. (2019). Revolución eléctrica en Everis Perú (Ed.), Transformación Digital en el Perú. Pentagraf.

Roca Zegarra, M. (2019). Brazo innovador en Everis Perú (Ed.), Transformación Digital en el Perú. Pentagraf.

Saldarriaga, J. (27 de 03 de 2020). Instituto de Ingenieros de Minas del Perú. Obtenido de Diario el Comercio: https://elcomercio.pe/economia/dia1/mineria-covid-19-pandemia-acelerara-la-transformacion-digital-de-lamineria-en-el-peru-pandemia-noticia/

Salesforce inc. (01 de 09 de 2017). ¿Qué es Transformación Digital? Obtenido de https://www.salesforce.com/br/blog/2017/Setembro/O-que-eTransformacao-Digital.html

Uccelli, M. (2019). Digitales y relevantes en Everis Perú (Ed.), Transformación Digital en el Perú. Pentagraf.

R. Andrés. (21 de 07 de 2020). UCl Un Canal Inteligente. Obtenido de http://uci.pe/2020/07/21/emolienteros-cobraran-con-tarjetas-para-prevenircontagios-de-coronavirus/

Wikipedia. (s.f.). Wikipedia. Obtenido de https://es.wikipedia.org/wiki/Transformaci\%C3\%B3n_digital 